\title{
LIMK1 and LIMK2 regulate cortical development through affecting neural progenitor cell proliferation and migration
}

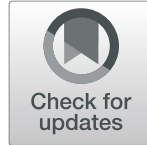

\author{
Rui Mao', Rui Deng', Yan Wei', Lifang Han', Yanghong Meng ${ }^{2}$, Wei Xie ${ }^{1 *}$ and Zhengping Jia ${ }^{1,2,3^{*}}$
}

\begin{abstract}
LIMK1 and LIMK2 are key downstream targets to mediate the effects of the Rho family small GTPases and p21activated kinases (PAK) in the regulation of the actin cytoskeleton. LIMKs are also critical for synaptic transmission, plasticity and memory formation. Changes in LIMK signaling are associated with several neurodevelopmental and neurodegenerative diseases, including autism, intellectual disability and Alzheimer's disease. However, the role of LIMK signaling in brain development remains unknown. In this study, we used LIMK1 KO and LIMK2 KO mice to investigate the role of LIMK signaling in the cerebral cortical development. We found that these KO mice are reduced in the number of pyramidal neurons in upper cortical layers and this reduction is accompanied by a smaller pool of neural progenitor cells and impaired neuronal migration. These results are similar to those found in PAK1 KO mice and suggest that LIMK-dependent actin regulation may play a key role in mediating the effects of PAK1 and Rho signaling in the regulation of cortical development.
\end{abstract}

Keywords: Cortical development, LIMK1, LIMK2, Neuronal proliferation, Migration, Actin

\section{Introduction}

Mammalian neocortex is a major brain area that is critical for various brain functions, including cognition and sensory perception [1-3]. Thus, the precise assembly of neocortex is an essential component of brain development. Abnormities in generation, differentiation or migration of neurons, key processes involved in neocortical development, can cause various neurological and mental disorders, including microcephaly, autism and intellectual disability [4-6]. Although many molecules and signaling pathways such as Reelin and Notch have been reported to be involved, the exact mechanisms underlying precise cortical development remain unclear [7-10]. The actin cytoskeleton is a major structural component of the cell and is required for cell morphology and migration, but its role in mouse brain development is poorly understood [11]. We have previously shown that p21-activated kinase 1 (PAK1), a key target of the Rho family small GTPases

\footnotetext{
* Correspondence: wei.xie@seu.edu.cn; zhengping.jia@sickkids.ca

${ }^{1}$ The Key Laboratory of Developmental Genes and Human Disease, Ministry of Education, Institute of Life Sciences, Southeast University, 2 Sipailou Road, Nanjing 210096, China

Full list of author information is available at the end of the article
}

essential for actin reorganization, regulates neocortical development by promoting the proliferation of neural progenitor cells and neuronal migration in the cortex [12]. But because PAK1 can target multiple processes in addition to the actin cytoskeleton [13-15], whether the effect of PAK1 on cortical development is mediated by actin remains unknown.

LIM-domain containing kinase 1 and 2 (LIMK1 and LIMK2) are a family of serine/threonine protein kinases that are critical for actin regulation [16-18]. Both LIMK1 and LIMK2 can directly phosphorylate and inactivate the actin depolymerization factor (ADF)/cofilin and promote assembly of actin filaments (F-actin) [19, 20]. This ADF/ cofilin-dependent process represents a key mechanism by which the Rho family small GTPase and PAKs regulate actin reorganization [21]. Using knockout mice, we have previously shown that both LIMK1 and LIMK2 are involved in neuronal growth and morphology, synaptic function, pain response and learning and memory [22-26]. However, exactly how LIMK1/2 regulate these processes remain unclear. Because LIMK1/2 are expressed in developing brain $[17,27,28]$, we hypothesize that LIMK1/2 may play a role in the development of the brain. In this study, 
we investigated the role of LIMK1/2 in mouse neocortical development using LIMK1 KO, LIMK2 KO and LIMK1/2 double KO (DKO) mice. We show that these KO mice are significantly altered in neural proliferation, differentiation and neuronal migration in developing neocortex compared to wild type (WT) littermates. These alterations are similar to those observed in PAK1 KO mice [12]. These results provide strong evidence that LIMK/cofilin dependent actin regulation is a major factor in neocortical development. Because LIMK1 is genetically linked to a number of neurological and mental disorders [18], the present study suggests that LIMK1-dependent abnormalities in brain development may contribute to these disorders.

\section{Results}

Reduced cortical neurons in LIMK KO mice

Although LIMK1 and LIMK2 show significant structural similarities, their protein expression, subcellular localization and functions are considerably different [29]. Therefore, it is possible that LIMK1 and LIMK2 may play different roles in cortical development. To investigate this possibility, we first detected the expression of LIMK1 and LIMK2 in mouse brain from embryonic day to adult. The result of western blot and immunofluorescent staining confirmed that both LIMK1 and LIMK2 are expressed in developing embryonic brain (Fig. 1). Consistent with previous reports, LIMK1 was mainly localized in the neurites while isoforms of LIMK2 differentially expressed at diverse developmental stage and show different subcellular localization [17, 30, 31]. We then analyzed the overall cellular organization of the cortex of LIMK1 KO, LIMK2 KO, LIMK1/2 DKO and their WT littermates using layer-specific markers. The P0 pups were used since the production of cortical neuron have finished by this time. As shown in Fig. 2, the distribution of the layer-specific markers was similar in all genotypes, indicating that the overall laminar organization of the cortex was not grossly altered in these mice (Fig. 2). However, LIMK KO mice showed reduced late born neurons $\left(\mathrm{Brn}^{+}\right)$compared
A

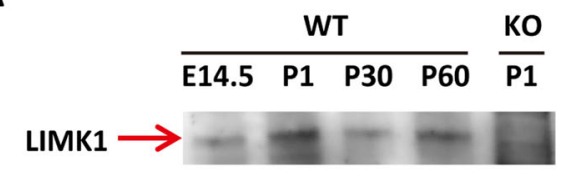

GAPDH

C

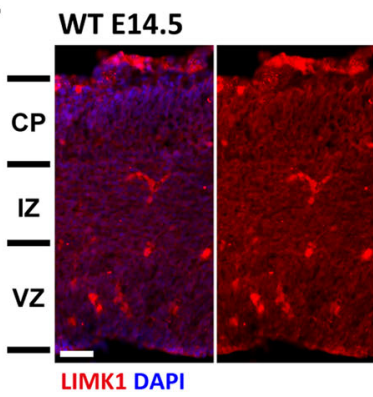

$\mathbf{F}$

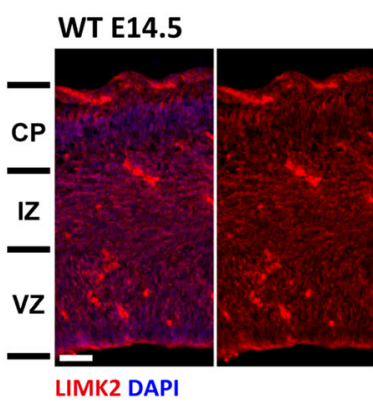

D WT P30

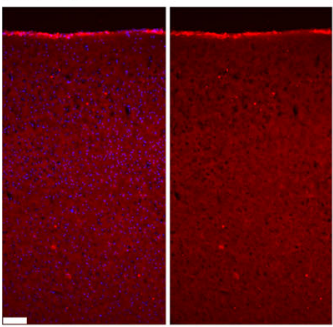

LIMK1 DAPI

G
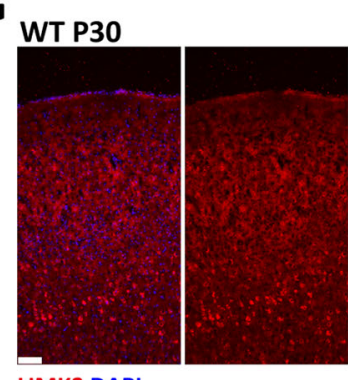

B

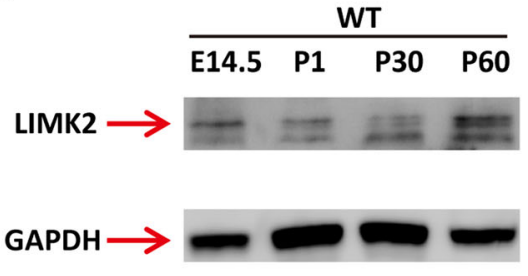

E

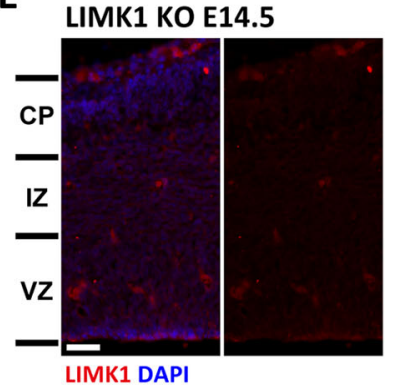

H

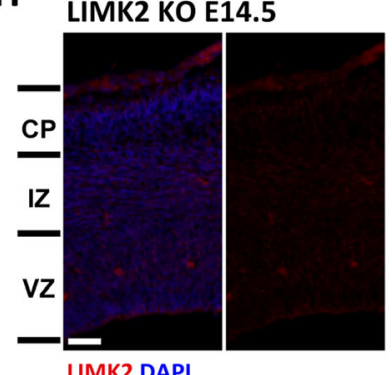

Fig. 1 Expression of LIMK1 and LIMK2 in developing mouse neocortex. a, b Western blots showing LIMK1 (a) and LIMK2 (b) expression in mouse neocortex at various developmental stages. (c, $\mathbf{d}$, e) Coronal brain sections co-stained with anti-LIMK1 and DAPI showing the expression of LIMK1 in WT E14.5 (c) and WT P30 (d), but not in LIMK1 KO E14.5 (e). (f, $\mathbf{g}, \mathbf{h})$ Coronal brain sections co-stained with anti-LIMK2 and DAPI on coronal brain sections of WT E14.5 (f) and WT P30 (g), but not in LIMK2 KO E14.5 (h). Note some remaining staining signals in LIMK1 and LIMK2 KO sections, suggesting that the antibodies might be non-specific. CP, cortical plate; IZ, intermediate zone; VZ, ventricular zone. Scale bars, $50 \mu \mathrm{m}$ 

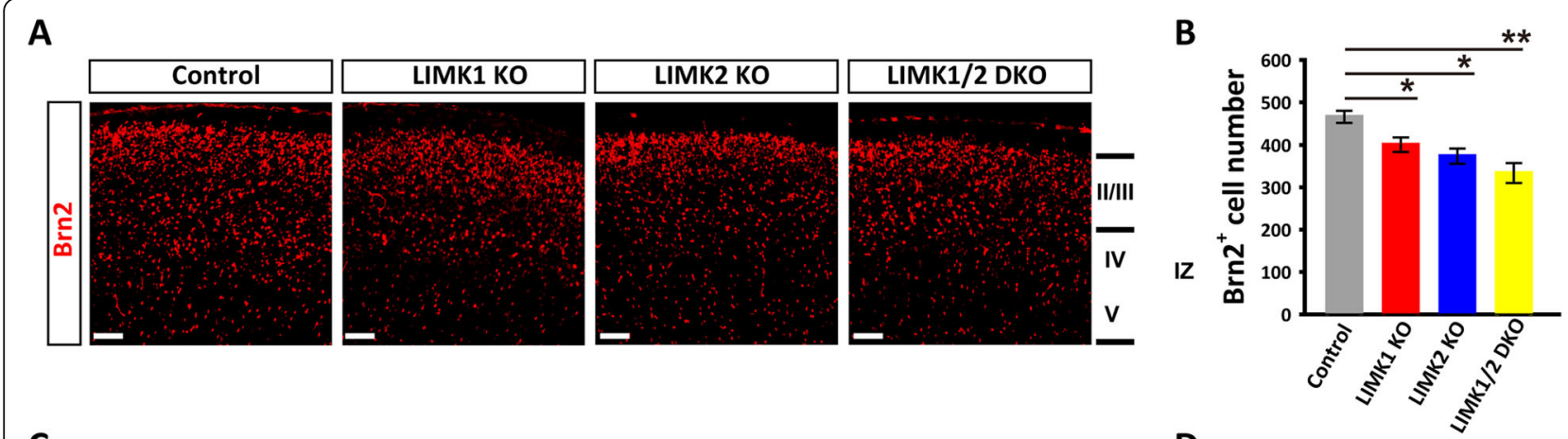

C
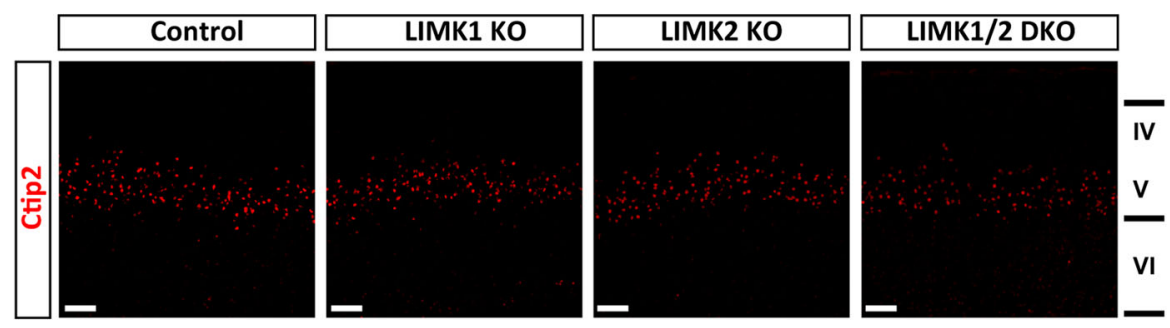

E
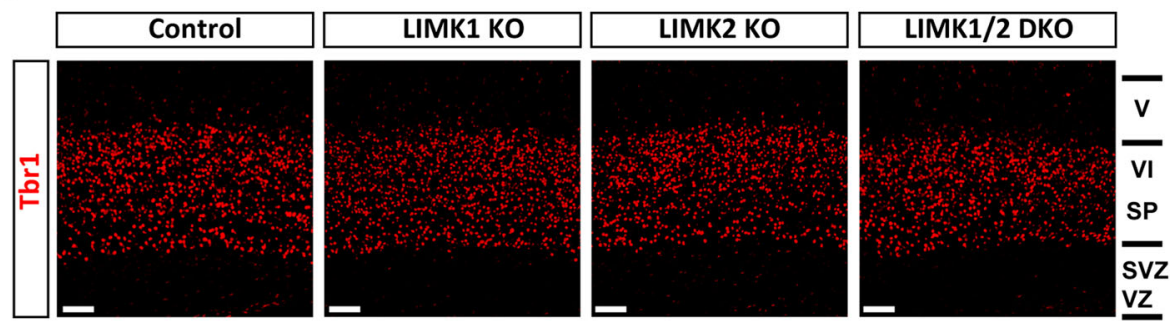

$\mathbf{F}$
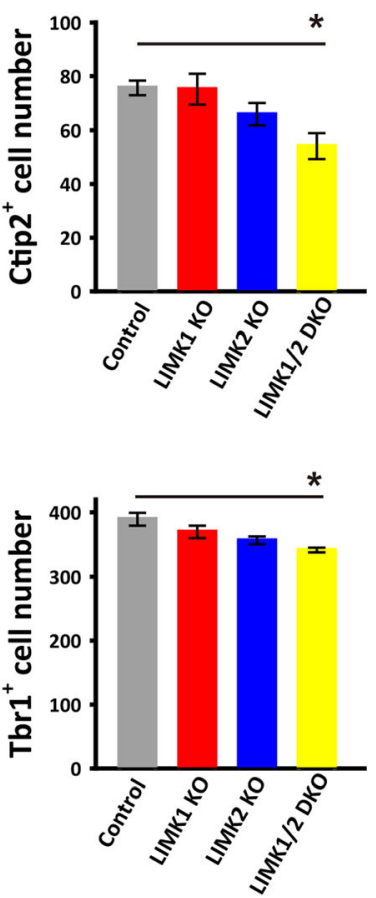

Fig. 2 Altered layer-specific markers in LIMK1 KO and LIMK2 KO cortex. a, b Sample coronal brain sections of postnatal day 0 (P0) stained for the late-born neuronal marker Brn2 (layer II and III) and summary graph showing reduced Brn2 ${ }^{+}$neurons in LIMK2 KO and LIMK1/2 DKO mice. c and d Sample coronal brain sections stained for the layer V neuronal marker Ctip2 and summary graph showing reduced Ctip2 $2^{+}$neurons in LIMK1/2 DKO mice. e and $\mathbf{f}$ Sample coronal brain sections stained for the layer VI neuronal marker Tbr1 and summary graph showing a trend of reduction in Tbr $1^{+}$neurons in LIMK2 KO and LIMK1/2 DKO mice. WT $n=7$, LIMK1 KO $n=6$, LIMK2 KO $n=6$, LIMK1/2 DKO $n=5$. Data are presented as mean \pm SEM. ${ }^{*} p<0.05,{ }^{* *} p<0.01,{ }^{* * *} p<0.001$, one-way ANOVA, Dunnett's test. Scale bars, $50 \mu \mathrm{m}$

to WT littermates (Fig. 2a and b). In addition, the Ctip2 ${ }^{+}$ and $\mathrm{Tbr} 1^{+}$neurons in the middle layers were also reduced in LIMK1/2 DKO mice (Fig. 2c-f). These data indicate that LIMKs are important for normal cortical development.

\section{Altered cortical distribution of $\mathrm{BrdU}^{+}$neurons in LIMK KO mice}

Having known that the late born pyramidal neurons were decreased in LIMK1 KO, LIMK2 KO and LIMK1/2 DKO mice, we then asked whether the loss of late born pyramidal neurons was a result of impaired neural migration and/or proliferation. We injected pregnant mice with BrdU at E14.5, a time window when the late born pyramidal neurons (laye II and layer III) are generated and migrating, to label the proliferating neural progenitors cells and their progeny. We first analyzed the number of BrdU labeled Brn2 $2^{+}$neurons at P0. Compared to control mice, the number of $\mathrm{BrdU}^{+} / \mathrm{Brn}^{+}$neurons was decreased in LIMK1 KO, LIMK2KO and LIMK1/2 DKO mice (Fig. 3). We also analyzed the distribution of all $\mathrm{BrdU}^{+}$neurons across the cortical plate. The neocortex was divided into equal 10 bins and the percentage of $\mathrm{BrdU}^{+}$neurons in each bin was quantified. As shown in Fig. 4, the total number of $\mathrm{BrdU}^{+}$neurons was significantly lower in LIMK1 KO, LIMK2 KO and LIMK1/2 DKO mice compared to WT control (Fig. $4 \mathrm{a}, \mathrm{b}$ ). It is important to note that although the total number of $\mathrm{BrdU}^{+}$ neurons was reduced, their fractions in the VZ/SVZ area 
A
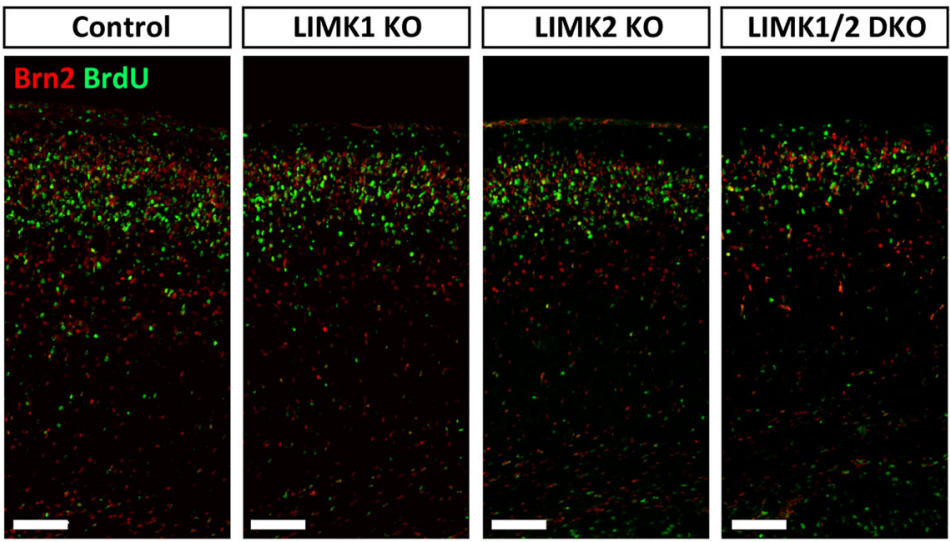

B
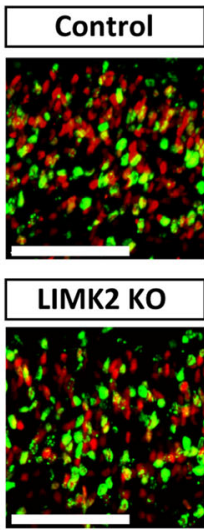
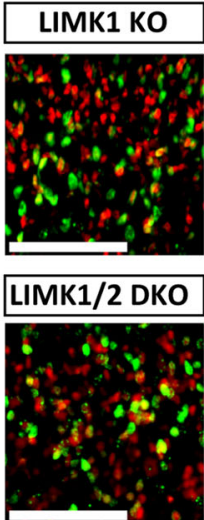

C

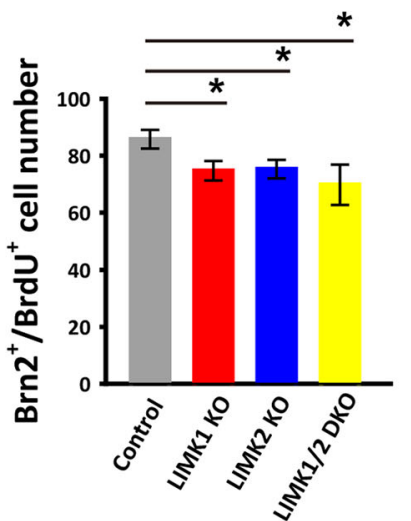

Fig. 3 Reduced pyramidal neurons in the upper cortical layer of LIMK KO mice. $\mathbf{a}$ and $\mathbf{b}$ Sample coronal brain sections stained for the late-born neuronal marker Brn2 (red) and BrdU (green) injected at E14.5, showing co-location of Brn2 ${ }^{+}$and BrdU ${ }^{+}$neurons (yellow) in the upper layer of P0 cortex. c Summary graph showing reduced Brn2 ${ }^{+} / \mathrm{BrdU}^{+}$neurons in LIMK1 KO, LIMK2 KO and LIMK1/2 DKO mice. WT $n=6$, LIMK1 KO $n=5$, LIMK2 KO $n=5$, LIMK1/2 DKO $n=3$. Data are presented as mean \pm SEM. ${ }^{*} p<0.05$, one-way ANOVA, Dunnett's test. Scale bars, $50 \mu m$

were significant greater compared to WT control (Fig. 4a and c). These results suggest that LIMK1/2 are involved in both neural proliferation and neuronal migration.

Impaired neural progenitor cell proliferation in LIMK KO mice To further investigate the nature of the reduced $\mathrm{BrdU}^{+}$ neurons in LIMK KO mice, we injected the pregnant mice at E14.5 with a high concentration of BrdU (200 $\mathrm{mg} / \mathrm{kg}$ ) for a brief period of time (i.e., $2 \mathrm{~h}$ ) and the brains of the embryos were then immediately fixed and sectioned for BrdU staining. This allowed an estimate of the rate of neural progenitor cell proliferation at this particular time. As shown in Fig. 5a-c, the number of BrdU ${ }^{+}$ proliferating progenitors was significantly reduced in LIMK1 KO, LIMK2 KO and LIMK1/2 DKO mice compared to WT mice, suggesting that both LIMK1 and LIMK2 are important for cell proliferation. To determine whether there were alterations in cell death, we performed the TUNEL staining for apoptotic cells. As shown in Fig. 5d and e, increased staining was observed in the dorsal telencephalon of LIMK2 KO and LIMK1/2 DKO mice, but not in LIMK1 KO mice. These results suggest that although both LIMK1 and LIMK2 are involved in neural proliferation, only LIMK2 may play a role in embryonic cell apoptosis.

\section{Reduced neural progenitors in LIMK KO mice}

To further determine the identity of the BrdU ${ }^{+}$cells in VZ/SVZ area, we performed immunostaining for Pax6 (a marker for self-renewal neural progenitor cells) and Tbr2, (a marker for intermediate progenitors) on the E14.5 brain sections. As shown in Fig. 6, while the number of $\mathrm{Pax}^{+}$progenitors showed no changes in any of the LIMK KO mice, the Tbr2 ${ }^{+}$progenitors were significantly reduced in LIMK1 KO, LIMK2 KO and LIMK1/2 DKO mice compared to WT control. These results suggest that LIMKs regulate neural progenitor cells primarily through affecting intermediate progenitors. 
A

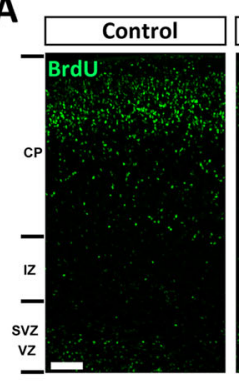

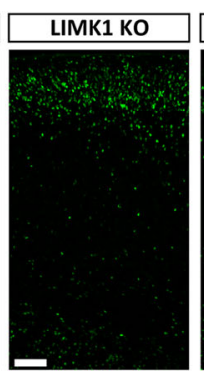
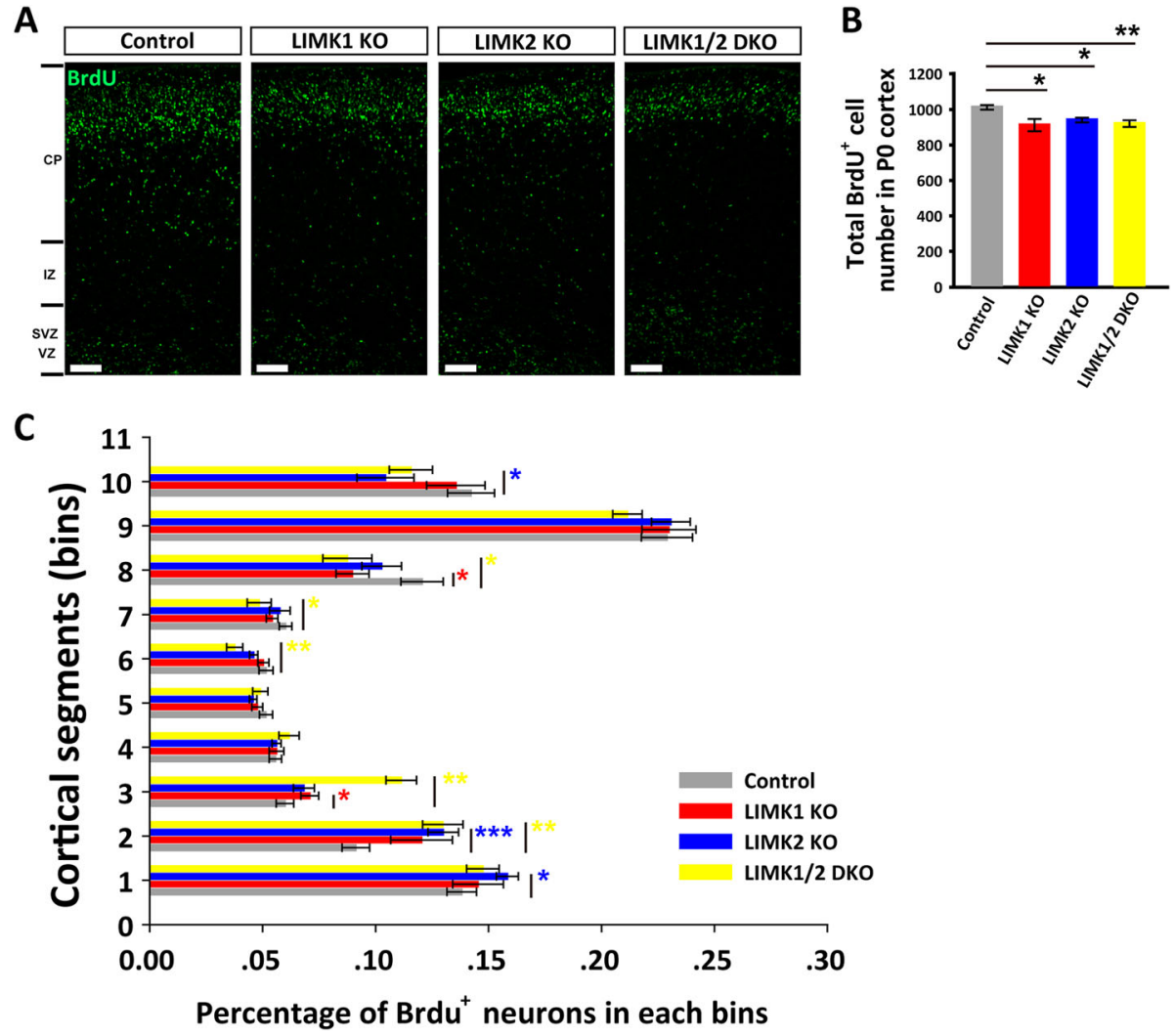

Fig. 4 Impaired neuronal migration in in LIMK KO mice. a Pregnant mice were labeled with BrdU at E14.5 and postnatal day 0 (P0) pups were stained for BrdU showing reduced BrdU ${ }^{+}$cells in LIMK1 KO, LIMK2 KO and LIMK1/2 DKO mice. b Summary graph showing reduced total BrdU cells in LIMK1 KO, LIMK2 KO and LIMK1/2 DKO mice. c Distribution of BrdU ${ }^{+}$cells across the cortex from (a), 1-10 represent the spatial order from VZ/SVZ to CP. WT control, $n=6$; LIMK1 KO, $n=5$; LIMK2 KO, $n=5$; LIMK1/2 DKO, $n=3$. Data are presented as mean \pm SEM. Statistical significance was determined by one-way ANOVA, Dunnett's test. ${ }^{*} p<0.05$, ** $p<0.01$. Scale bars, $50 \mu \mathrm{m}$

\section{LIMKs regulate cell cycle progression}

To investigate how LIMKs regulate neural proliferation, we examined the cell cycle state (active or inactive) of neural progenitor cells in LIMK KO mice. The pregnant mice were injected with $\mathrm{BrdU}(50 \mathrm{mg} / \mathrm{kg})$ at E13.5, and $24 \mathrm{~h}$ later the brain sections were co-stained for Ki67 (a marker for the active phases of the cell cycle) and BrdU. The data showed that the number of active proliferating cells $\left(\mathrm{BrdU}^{+}\right.$ and $\mathrm{Ki}^{+} \mathrm{7}^{+}$) was significantly decreased in LIMK2 KO and LIMK1/2 DKO mice (Fig. 7a-c). Accordingly, the number of cells that exited from the cell cycle $\left(\mathrm{BrdU}^{+}\right.$and $\left.\mathrm{Ki}^{-} 7^{-}\right)$ was increased in LIMK2 KO and LIMK1/2 DKO mice (Fig. $7 \mathrm{a}-\mathrm{d})$. In addition, the total number of $\mathrm{Ki}^{+} 7^{+}$cells was decreased in all three LIMK KO mice (Fig. 7e). To further investigate whether proliferation of radial glia cells and intermediate progenitor cells were affected differently, we co-stained Sox 2 and Tbr2 in BrdU incorporated sections. The result showed that only the number of intermediate progenitor cells $\left(\mathrm{Tbr} 2^{+} / \mathrm{BrdU}^{+}\right)$was significantly decreased (Fig. 8). These results suggested that LIMKs are particularly important in maintaining the proliferating capacity of the intermediate progenitor cells.

\section{Discussion}

It is well established that LIMK 1 and 2 are key players in the regulation of the actin cytoskeleton through phosphorylation and inactivation of cofilin [18-20,27]. Given the critical importance of the actin cytoskeleton in various cellular functions, particularly neuronal morphology and motility, it is not surprised that genetic ablation of the LIMK1/2 caused profound impairments in neuronal structure and function, including axonal and spine morphology, synaptic plasticity and memory in adult mice [22-26]. However, whether LIMK1/2 are also involved in brain development remains unknown. In this study, we investigated the role of LIMK1/2 in mouse neocortical development by examining neural progenitor cell proliferation, neuronal migration and neocortical layer organization in developing LIMK1 KO, LIMK2 KO and LIMK1/2 DKO mice. We showed that these KO mice exhibit significant deficits in both neural proliferation and migration, suggesting that LIMK-mediated actin is a critical player in these processes. In addition, we show that LIMK1 KO and LIMK2 KO mice are differentially altered in cell proliferation and apoptosis, 


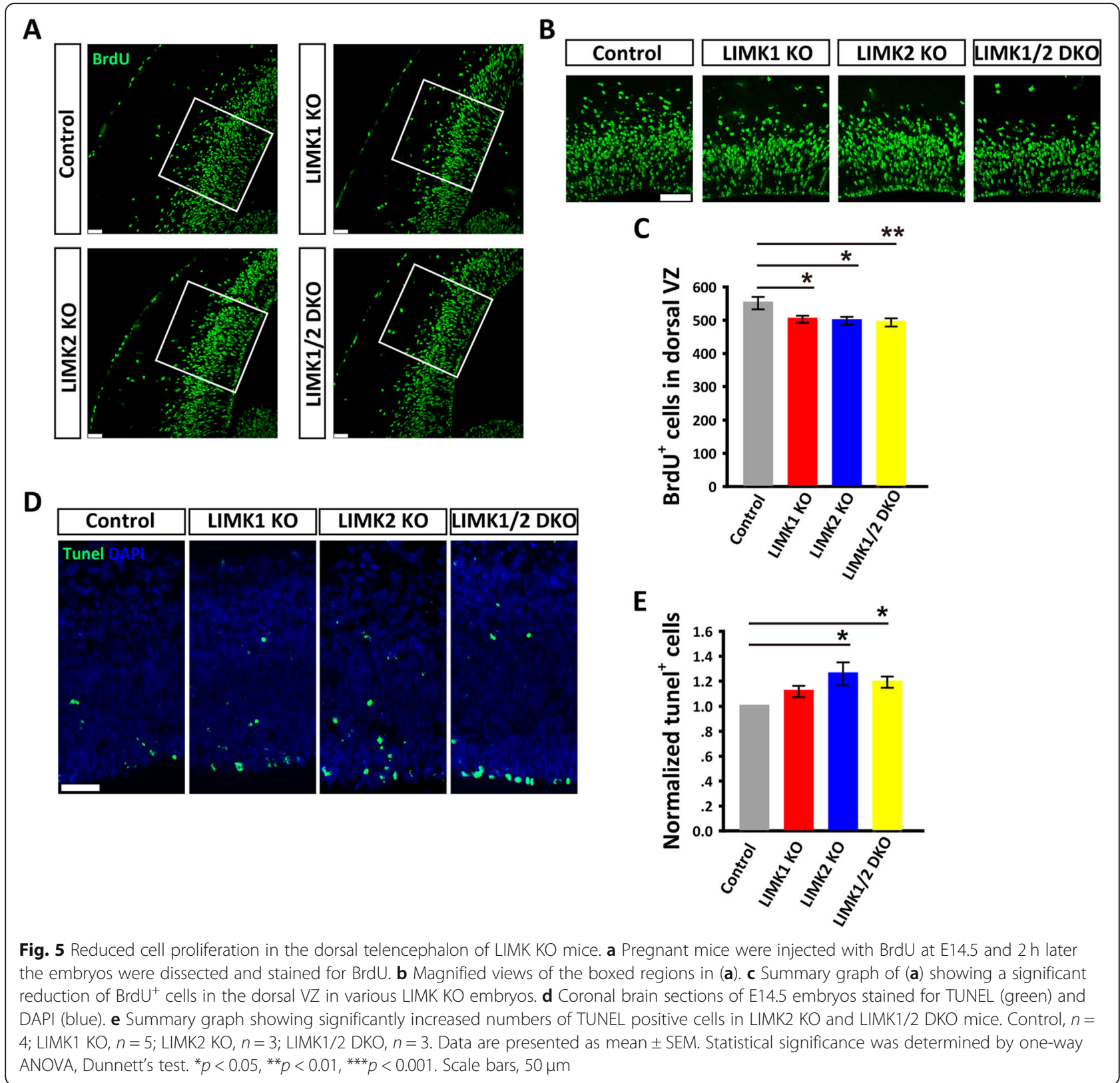

suggesting that LIMK1 and LIMK2 may play distinct roles in some aspects of brain development. Our results provide strong in vivo evidence for the critical involvement of LIMK signaling and actin in embryonic brain development.

The initiation of neuronal migration is activated by the of membrane receptors through local environmental cues followed by the polarization of the actin cytoskeleton and the extension of the leading process of the cell $[32,33]$. As key regulators of actin dynamics, LIMKs have been proved to play regulatory role in cell motility, particularly in tumor cell invasion, including human breast cancer cell lines and Jurkat $\mathrm{T}$ cells [34-36]. In contrast, their function in neuronal migration remains unknown. In this study, we demonstrated that the upper layer pyramidal neurons are reduced in LIMK1/2 KO mice compare to the WT littermates. These results are similar to those observed in PAK1 KO mice [12], suggesting that LIMKs are downstream targets of PAK1 in the regulation of cortical neuronal migration.

We also find that LIMK KO mice are impaired in the proliferation of neural progenitor cells, which is similar to the deficits reported in the PAK1 KO mice [12]. In LIMK KO mice, the number of progenitor cells at E14.5, especially the intermediate progenitors, is significantly less than that in control mice. Previous studies in 

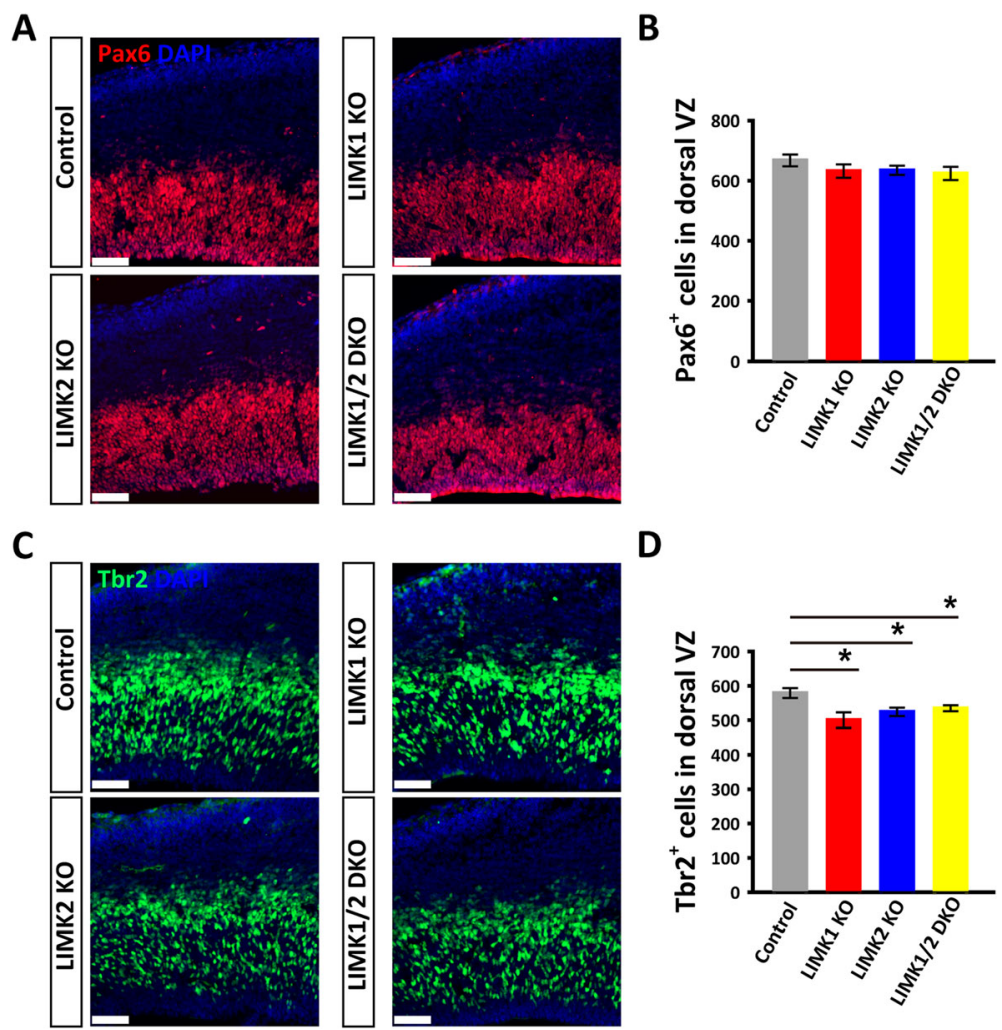

Fig. 6 Coronal brain sections of WT control and LIMK KO mice E14.5 embryos were stained for Pax6 or Tbr2. a Pax6 (red) and DAPI (blue) staining of WT control and various LIMK KO mice. b Summary graph of Pax6 positive cells showing no differences between control and various LIMK KO mice. c Tbr2 (green) and DAPI (blue) staining of WT control and various LIMK KO mice. d Summary graph showing significantly decreased numbers of Tbr2 positive cells in LIMK1 KO, LIMK2 KO and DKO mice compared to their control littermates. Control, $n=6$; LIMK1 KO, $n=3$; LIMK2 $\mathrm{KO}, n=5 ; \mathrm{LIMK} 1 / 2 \mathrm{DKO}, n=3$. Data are presented as mean $\pm \mathrm{SEM}$. Statistical significance was determined by one-way ANOVA, Dunnett's test. ${ }^{*} p<0.05,{ }^{* *} p<0.01$. Scale bars, $50 \mu \mathrm{m}$

cultured cell lines have shown that LIMKs participate in several steps of cell cycle progression [37-39]. LIMK1 and LIMK2 have distinct subcellular localization and play different roles during cell division [40, 41]. Inhibition of LIMK1 activity during mitosis causes activation of cofilin and lead to delayed transition from metaphase to anaphase and irregular spindle positioning, while LIMK2 is important for normal mitotic spindle formation [38, 39, 42]. During cortical development, neural progenitor fata decision and neurogenesis is closely associated with asymmetric and symmetric cell division, when the accurate spindle positioning and orientation is essential for cells to determine the fates of daughter cells after mitosis $[43,44]$. In addition, the length of cell cycle is one of the main elements that affect the final output of total neurons [45-47]. The differential role played by LIMK1 and LIMK2 in spindle positioning and formation, respectively, may have different effects on cell division and cell death. This may be responsible for specific deficits in LIMK2 KO mice where we observed increased apoptotic cell death, which is not detected in LIMK1 or PAK1 KO mice. Our results are consistent with a previous study showing that LIMK2 knockdown in neuroblastoma cells increased the sensitivity to microtubule-targeted drugs and caused enhanced apoptosis [38]. Our results that KO mice for LIMK1, LIMK2 or PAK1 have similarities and difference in changes in embryonic neuronal proliferation and cell cycle progression provide in vivo evidence that they have both shared and distinct functions in these processes.

In conclusion, we show here that LIMK1 KO and LIMK2 KO mice are impaired in neuronal migration and progenitor cell proliferation with some changes unique to one or the other $\mathrm{KO}$ mice. These impairments are similar to those found in PAK1 KO mice and suggest that LIMK-dependent actin reorganization plays a key role in mediating the effects of PAK1 in the regulation of the cortical development.

\section{Methods}

\section{Animals}

The generation and initial characterization of LIMK1 and LIMK2 KO mice were described previously $[23,48]$. The LIMK- $1 / 2$ DKO mice were 
A
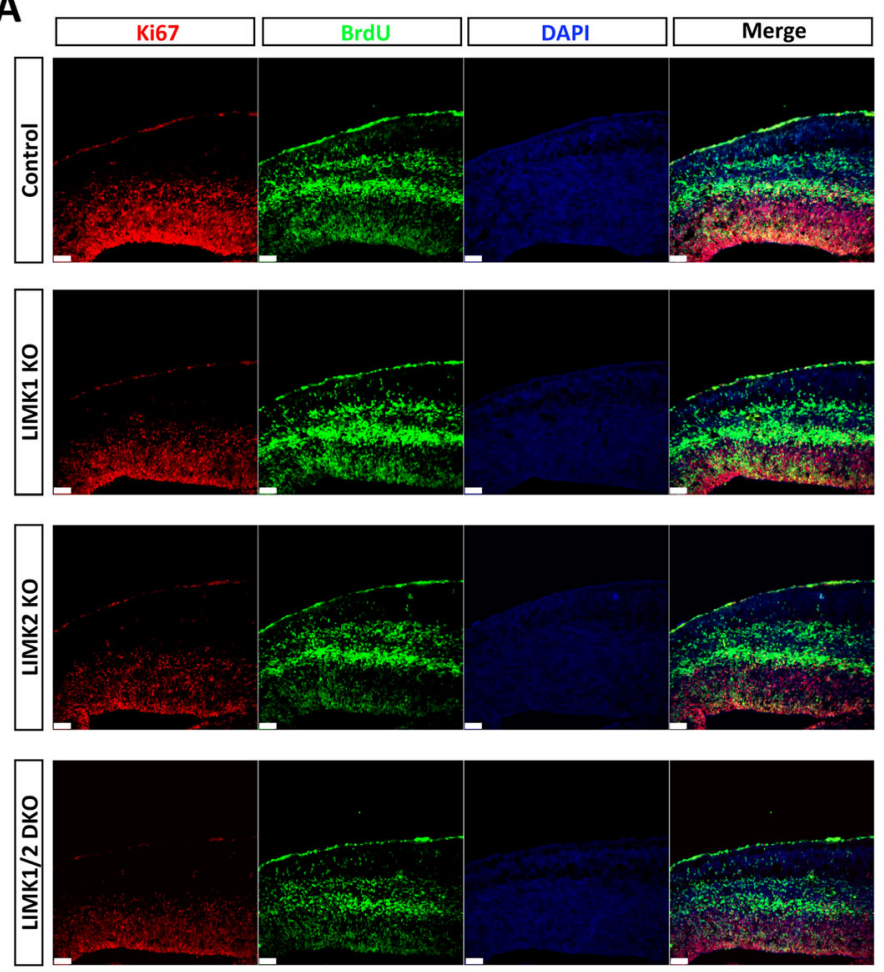

C

D
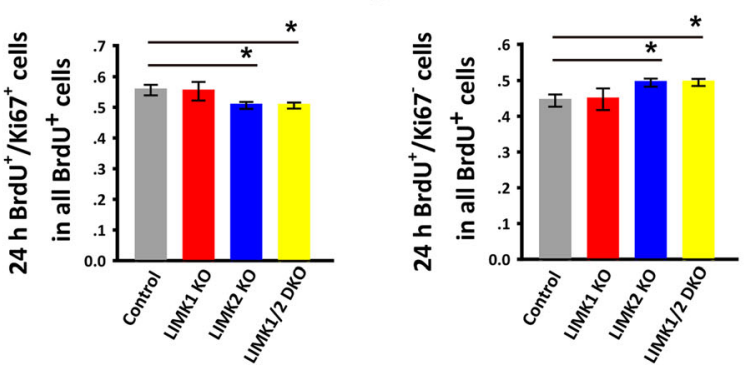

B
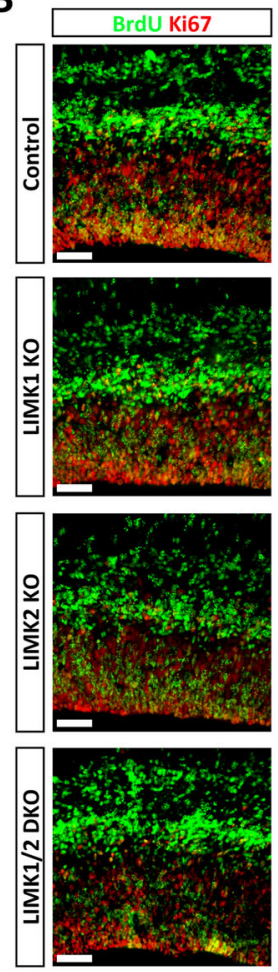

$\mathbf{E}$

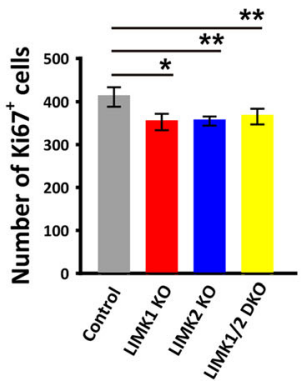

Fig. 7 Altered cell cycle state in the telencephalon of LIMK KO mice. a Pregnant mice were injected with BrdU at E13.5 for $24 \mathrm{~h}$ and the embryos of the control and various LIMK KO were then dissected at E14.5 and processed for immunostaining for BrdU (green), Ki67 (red) and DAPI (blue). b Magnified views of the co-localization of $\mathrm{BrdU}^{+} / \mathrm{Ki}^{6} 7^{+}$cells. c-e Summary graphs of the percentages of $\mathrm{BrdU}^{+} / \mathrm{Ki}^{6} 67^{+}(\mathbf{c}) \mathrm{and} \mathrm{BrdU}^{+} / \mathrm{Ki}^{6} 67^{-}(\mathbf{d}) \mathrm{cells}$ relative to the total $\mathrm{BrdU}^{+}$cells showing a significantly decreased number of cells staying in the active phases of the cell cycle, as indicated by reduced $\mathrm{BrdU}^{+} / \mathrm{Ki}_{6} 7^{+}$cells and increased $\mathrm{BrdU}^{+} / \mathrm{Ki} 67^{-}$cells, in LIMK KO mice compared to the control littermates. Summary graph showing that the number of total Ki67 $7^{+}$cells (e) was also decreased in the dorsal telencephalon in the LIMK KO mice compared to the control littermates. Control, $n=6$; LIMK1 KO, $n=3$; LIMK2 KO, $n=5$; LIMK1/2 DKO, $n=3$. Data are presented as mean \pm SEM. Statistical significance was determined by one-way ANOVA, Dunnett's test. ${ }^{*} p<0.05,{ }^{* *} p<0.01$. Scale bars, $50 \mu \mathrm{m}$

obtained from LIMK-1 +/- LIMK-2 +/- interbreeding. To minimize the effect of genetic background, all the pups and embryos used in this study were LIMK1/2 KOs and their WT or heterozygotes littermates derived from the heterozygous breeders. All the mice were housed under a standard 12-h light/12-h dark cycle condition at the Experimental Animal Center at Southeast University, China. All animal experiment procedures were approved by Southeast University (Nanjing, China) and the Hospital for Sick Children (Toronto, Canada) Animal Care and Use Committee.

\section{Tissue processing and immunohistochemistry}

Procedures for brain processing and immunohistochemistry were described previously [12]. Briefly, for embryonic 14.5 (E14.5) samples, the pregnant mice were anesthetized by intraperitoneal injection with $7 \%$ chloral hydrate $(10 \mu \mathrm{l} / \mathrm{g})$. The fetal brains were dissected and fixed with $4 \%$ paraformaldehyde (PFA) for $6 \mathrm{~h}$ at $4{ }^{\circ} \mathrm{C}$, and PFA was replaced with $30 \%$ sucrose dissolved in PBS at $4{ }^{\circ} \mathrm{C}$ until the brains were saturated. For postnatal samples, the neonatal mice were anaesthetized by hypothermia, then subjected to cardiac perfusion with 
A
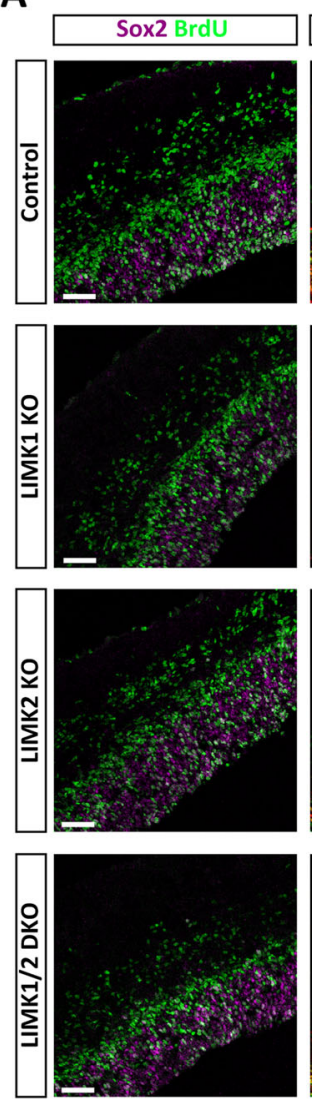
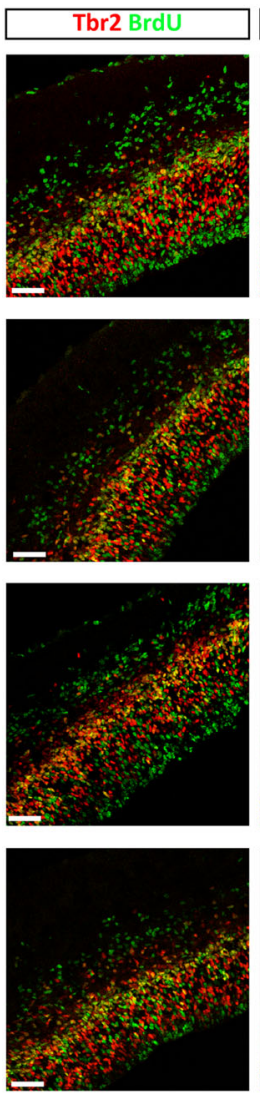
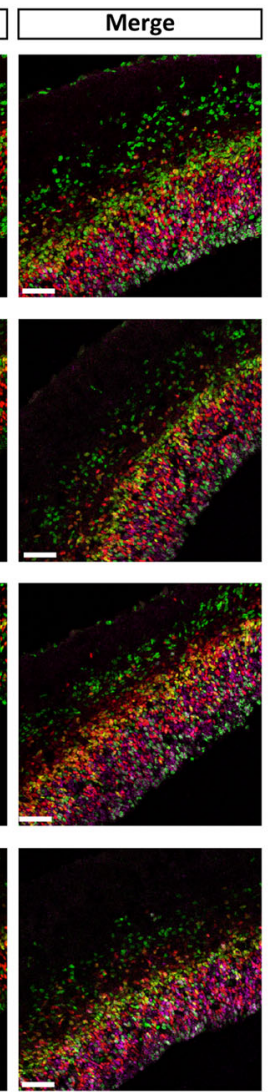

B

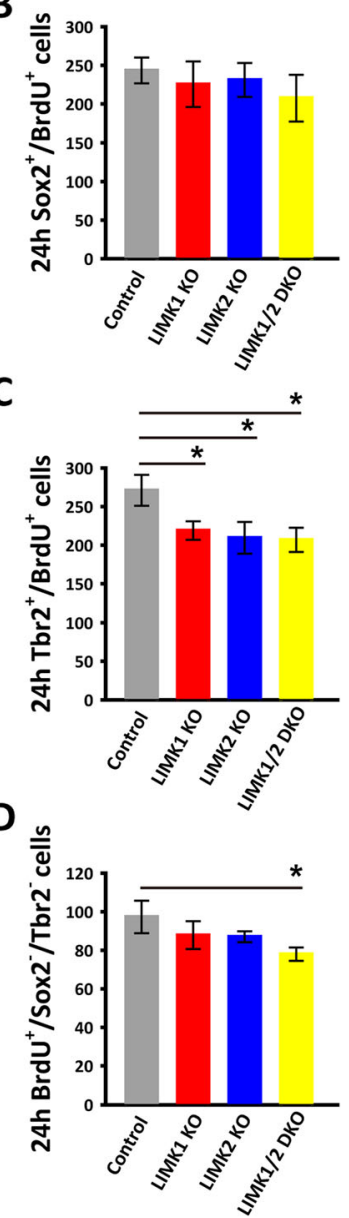

Fig. 8 Reduced proliferation of intermediate neural progenitors in LIMK KO mice. a Pregnant mice were injected with BrdU at E13.5 for $24 \mathrm{~h}$ and the embryos of the control and various LIMK KO were then dissected at E14.5 and processed for immunostaining for Sox2 (purple), Tbr2 (red) and $\mathrm{BrdU}$ (green). b-d Summary graphs of the number of $\mathrm{Sox2}^{+} / \mathrm{BrdU}^{+}(\mathbf{b}), \mathrm{Tbr}^{+} / \mathrm{BrdU}^{+}(\mathbf{c})$ and $\mathrm{BrdU}^{+} / \mathrm{Sox} 2^{-} / \mathrm{Tbr} 2^{-}$cells (d).Although the numbers of newborn radial glial cell $\left(\mathrm{Sox} 2^{+} / \mathrm{BrdU}^{+}\right)$, intermediate progenitor $\left(\mathrm{Tbr} 2^{+} / \mathrm{BrdU}^{+}\right)$and neurons $\left(\mathrm{BrdU}^{+} / \mathrm{Sox} 2^{-} / \mathrm{Tbr2} 2^{-}\right.$cells) all showed a trend of decrease in LIMK KO mice compared to the control littermates, only the reduction of intermediate progenitors $\left(\mathrm{Tbr}^{+} / \mathrm{BrdU}^{+}\right)$showed a significant difference. Control, $n=6$; LIMK1 KO, $n=3$; LIMK2 KO, $n=5$; LIMK1/2 DKO, $n=3$. Data are presented as mean \pm SEM. Statistical significance was determined by one-way ANOVA, Dunnett's test. ${ }^{*} p<0.05,{ }^{* *} p<0.01$. Scale bars, $50 \mu \mathrm{m}$

$0.1 \mathrm{M}$ phosphate buffered saline (PBS), followed by $4 \%$ PFA. The brains were dissected and fixed with $4 \%$ PFA overnight at $4{ }^{\circ} \mathrm{C}$. The PFA was replaced with $30 \%$ sucrose dissolved in PBS at $4{ }^{\circ} \mathrm{C}$ until the brains were saturated. The saturated brains were embedded in Tissue$\mathrm{Tek}^{\circ}$ O.C.T. Compound and frozen by liquid nitrogen. The samples were cut into coronal sections of $10 \mu \mathrm{m}$ for prenatal day brains and $16 \mu \mathrm{m}$ for postnatal day brains using a Leica CM1950 cryostat. Brain sections were washed in PBS for $10 \mathrm{~min}$, permeabilized with $0.1 \%$ Triton X-100 in PBS, blocked with $0.1 \%$ Triton X-100, 10\% fetal bovine serum in PBS for $2 \mathrm{~h}$ at room temperature, then incubated with primary antibodies overnight at $4{ }^{\circ} \mathrm{C}$. Subsequently, sections were washed in $0.1 \%$ PBT and incubated in appropriate secondary antibodies for $2 \mathrm{~h}$ at $37^{\circ} \mathrm{C}$.

\section{Antibodies and reagents}

Primary antibodies used in this study include: rabbit anti-Brn2 (Santa Cruz, 1:50), rabbit anti-Ctip2 (Abcam, 1:1000), rabbit anti-Tbr1 (Proteintech Group, 1:500), rat anti-BrdU (Abcam, 1:1000), rabbit anti-Ki67 (Abcam, 1: 500), mouse anti-Pax6 (Cell Signaling Technology, 1: 200), rabbit anti-Pax6 (Proteintech Group, 1:1000). Secondary antibodies used include: Alexa Fluor 555 donkey anti-rabbit IgG (Invitrogen, 1:300), Alexa Fluor 488 donkey anti-rabbit IgG (Invitrogen, 1:300), Alexa Fluor 488 goat anti-mouse IgG (Proteintech Group, 1:300), Alexa Fluor 488 goat anti-rat IgG (Jackson Immuno Research, 1:300). Cell apoptosis was detected by TUNEL FITC Apoptosis Detection Kit (Vazyme, A111). Nuclei were counterstained with 4, 6-diamidino-2-phenylindole (DAPI; Cayman Chemical). 


\section{Microscope setups and image collection}

Immunostaining images were collected at room temperature using confocal laser microscopes (LSM700, Carl Zeiss) or a light microscope (CTR 5000; Leica) at 2,048 $\times 2,048$ pixels. The images were then analyzed with Zeiss Aim Image Browser software or Image J software. In postnatal experiments, greater than 3 brains of each genotype from at least 5 litters were used for analysis. At least 3 discontinuous coronal sections from somatosensory cortex from each brain were counted. For the migration study, $\mathrm{BrdU}^{+}$cells were measured in vertical strips with a $350 \mu \mathrm{m}$ width and the cortical slice images were equally divided into ten bins across the six-layered cortex. The regions of cortex were identified by layer specific markers and the DAPI staining. In embryonic studies, greater than 3 brains of each genotype from at least 6 litters were used for immunostaining experiments. No less than 4 discontinuous coronal sections of the medial cortex from each brain were analyzed for cell counts. For each section, an area of 300-400 $\mu \mathrm{m}$ wide with a length spanning either VZ/SVZ or the entire middle regions of the E14.5 telencephalon was analyzed. The labeled cells were manually counted use the Cell Counter plugin of Image J software.

\section{Statistical analysis}

One-way ANOVA, Dunnett's test was used to evaluate the data statistically, and when $P<0.05$, the differences were considered significant. And all data were presented as means \pm standard errors of the means (SEM).

\section{Abbreviations}

BrdU: Bromodeoxyuridine; Brn2: Pou3f2 (POU domain, class 3, transcription factor 2); Ctip2: Bcl11b (B cell leukemia/lymphoma 11 B); Pax6: Paired box 6; Tbr1: T-box, brain 1; TUNEL: Terminal-deoxynucleoitidyl Transferase Mediated Nick End Labeling

\section{Acknowledgments}

We thank Dr. Nakamura for LIMK2 KO mice. We are grateful to all members of Jia and Xie labs for their technical assistance and comments on the manuscript.

\section{Authors' contributions}

$\mathrm{ZJ}$ and RM designed the study; RM, RD, YW and LH performed the experiments. RM, LH, YM, WX analyzed the data, RM and ZJ wrote the paper. All authors read and approved the final manuscript.

\section{Authors' information}

$\mathrm{RM}, \mathrm{RD}, \mathrm{YW}, \mathrm{LH}$ are graduate students in the Key Laboratory of Developmental Genes and Human Diseases, MOE, Southeast University, Nanjing, China. WX is a Professor and Director of the Key Laboratory of Developmental Genes and Human Diseases, MOE, Southeast University, Nanjing, China. YM is a Research Associate in the Hospital for Sick Children. ZJ is a Professor and Senior Scientist in the Department of Physiology, University of Toronto, and Program in Neurosciences and Mental Health, the Hospital for Sick Children.

\section{Funding}

This work was supported by grants from the National Natural Science Foundation of China (91632201 and 31430035, WX), the Canadian Institutes of Health Research (CIHR, MOP119421, ZPJ; PJT155959), Canadian Natural Science and Engineering Research Council NSERC RGPIN 341498 RGPIN06295, ZPJ), NSFC and CIHR Joint Health Research Initiative Program (81161120543, WX and CCl117959, ZPJ), Brain Canada (ZPJ) and the Hospital for Sick Children Foundation (ZPJ).

\section{Availability of data and materials}

The data used in our study are available from the authors on reasonable request.

\section{Ethics approval}

All animal experiment procedures were approved by Southeast University (Nanjing, China) and the Hospital for Sick Children (Toronto, Canada) Animal Care and Use Committees.

\section{Consent for publication}

Not applicable.

\section{Competing interests}

The authors declare that they have no competing interests.

\section{Author details}

'The Key Laboratory of Developmental Genes and Human Disease, Ministry of Education, Institute of Life Sciences, Southeast University, 2 Sipailou Road, Nanjing 210096, China. ${ }^{2}$ Neurosciences \& Mental Health, The Hospital for Sick Children, 555 University Ave, Toronto, ON M5G 1X8, Canada. ${ }^{3}$ Department of Physiology, Faculty of Medicine, University of Toronto, 1 King's College Circle, Toronto, ON M5S 1A8, Canada.

Received: 26 March 2019 Accepted: 2 July 2019

Published online: 18 July 2019

\section{References}

1. Harris J, Tomassy GS, Arlotta P. Building blocks of the cerebral cortex: from development to the dish. Wiley Interdiscip Rev Dev Biol. 2015;4:529-44.

2. Lui JH, Hansen DV, Kriegstein AR. Development and evolution of the human neocortex. Cell. 2011;146:18-36.

3. Sun T, Hevner RF. Growth and folding of the mammalian cerebral cortex: from molecules to malformations. Nat Rev Neurosci. 2014;15:217-32.

4. Jayaraman D, Bae BI, Walsh CA. The genetics of primary microcephaly. Annu Rev Genomics Hum Genet. 2018;19:177-200.

5. Kast RJ, Levitt P. Precision in the development of neocortical architecture: from progenitors to cortical networks. Prog Neurobiol. 2019;175:77-95.

6. Su SC, Tsai LH. Cyclin-dependent kinases in brain development and disease. Annu Rev Cell Dev Biol. 2011;27:465-91.

7. Frotscher M. Role for Reelin in stabilizing cortical architecture. Trends Neurosci. 2010;33:407-14.

8. Lakoma J, Garcia-Alonso L, Luque JM. Reelin sets the pace of neocortical neurogenesis. Development. 2011;138:5223-34.

9. Matsunaga Y, Noda M, Murakawa H, Hayashi K, Nagasaka A, Inoue S, Miyata T, Miura T, Kubo Kl, Nakajima K. Reelin transiently promotes N-cadherindependent neuronal adhesion during mouse cortical development. Proc Natl Acad Sci U S A. 2017:114:2048-53.

10. Yang Z, Li PF, Chen RC, Wang J, Wang S, Shen Y, Wu X, Fang B, Cheng X, Xiong ZQ. ADAM10-initiated release of notch intracellular domain regulates microtubule stability and radial migration of cortical neurons. Cereb Cortex. 2017b;27:919-32.

11. Pollard TD, Cooper JA. Actin, a central player in cell shape and movement Science. 2009:326:1208-12

12. Pan $X$, Chang $X$, Leung $C$, Zhou Z, Cao F, Xie W, Jia Z. PAK1 regulates cortical development via promoting neuronal migration and progenitor cell proliferation. Mol Brain. 2015;8:36.

13. Bokoch GM. Biology of the p21-activated kinases. Annu Rev Biochem. 2003; $72: 743-81$.

14. Xia S, Zhou Z, Leung C, Zhu Y, Pan X, Qi J, Morena M, Hill MN, Xie W, Jia Z. p21-activated kinase 1 restricts tonic endocannabinoid signaling in the hippocampus. Elife. 2016;5.

15. Zhao ZS, Manser E. PAK family kinases: physiological roles and regulation. Cell Logist. 2012;2:59-68.

16. Bernard O. Lim kinases, regulators of actin dynamics. Int J Biochem Cell Biol. 2007:39:1071-6.

17. Foletta VC, Moussi N, Sarmiere PD, Bamburg JR, Bernard O. LIM kinase 1, a key regulator of actin dynamics, is widely expressed in embryonic and adult tissues. Exp Cell Res. 2004;294:392-405.

18. Scott RW, Olson MF. LIM kinases: function, regulation and association with human disease. J Mol Med (Berl). 2007;85:555-68. 
19. Arber S, Barbayannis FA, Hanser H, Schneider C, Stanyon CA, Bernard O, Caroni P. Regulation of actin dynamics through phosphorylation of cofilin by LIM-kinase. Nature. 1998;393:805-9.

20. Yang N, Higuchi O, Ohashi K, Nagata K, Wada A, Kangawa K, Nishida E, Mizuno K. Cofilin phosphorylation by LIM-kinase 1 and its role in Racmediated actin reorganization. Nature. 1998;393:809-12.

21. Bamburg JR. Proteins of the ADF/cofilin family: essential regulators of actin dynamics. Annu Rev Cell Dev Biol. 1999;15:185-230.

22. Meng Y, Takahashi H, Meng J, Zhang Y, Lu G, Asrar S, Nakamura T, Jia Z. Regulation of ADF/cofilin phosphorylation and synaptic function by LIMkinase. Neuropharmacology. 2004;47:746-54.

23. Meng Y, Zhang Y, Tregoubov V, Janus C, Cruz L, Jackson M, Lu WY, MacDonald JF, Wang JY, Falls DL, et al. Abnormal spine morphology and enhanced LTP in LIMK-1 knockout mice. Neuron. 2002;35:121-33.

24. Phan KD, Hazen VM, Frendo M, Jia Z, Butler SJ. The bone morphogenetic protein roof plate chemorepellent regulates the rate of commissural axonal growth. J Neurosci. 2010;30:15430-40.

25. Todorovski Z, Asrar S, Liu J, Saw NM, Joshi K, Cortez MA, Snead OC 3rd, Xie W, Jia Z. LIMK1 regulates long-term memory and synaptic plasticity via the transcriptional factor CREB. Mol Cell Biol. 2015;35:1316-28.

26. Yang $X$, He G, Zhang $X$, Chen L, Kong Y, Xie W, Jia Z, Liu WT, Zhou Z. Transient inhibition of LIMKs significantly attenuated central sensitization and delayed the development of chronic pain. Neuropharmacology. 2017; 125:284-94.

27. Acevedo K, Li R, Soo P, Suryadinata R, Sarcevic B, Valova VA, Graham ME, Robinson PJ, Bernard O. The phosphorylation of p25/TPPP by LIM kinase 1 inhibits its ability to assemble microtubules. Exp Cell Res. 2007:313:4091-106.

28. Mori T, Okano I, Mizuno K, Tohyama M, Wanaka A. Comparison of tissue distribution of two novel serine/threonine kinase genes containing the LIM motif (LIMK-1 and LIMK-2) in the developing rat. Brain Res Mol Brain Res. 1997;45:247-54.

29. Manetti F. LIM kinases are attractive targets with many macromolecular partners and only a few small molecule regulators. Med Res Rev. 2012;32:968-98.

30. Tastet J, Cuberos H, Vallee B, Toutain A, Raynaud M, Marouillat S, Thepault RA, Laumonnier F, Bonnet-Brilhault F, Vourc'h P, et al. LIMK2-1 is a Hominidae-specific isoform of LIMK2 expressed in central nervous system and associated with intellectual disability. Neuroscience. 2019;399:199-210.

31. Vallee B, Cuberos H, Doudeau M, Godin F, Gosset D, Vourc'h P, Andres CR, Benedetti H. LIMK2-1, a new isoform of human LIMK2, regulates actin cytoskeleton remodeling via a different signaling pathway than that of its two homologs, LIMK2a and LIMK2b. Biochem J. 2018;475:3745-61.

32. da Silva JS, Dotti CG. Breaking the neuronal sphere: regulation of the actin cytoskeleton in neuritogenesis. Nat Rev Neurosci. 2002;3:694-704.

33. Pollard TD, Borisy GG. Cellular motility driven by assembly and disassembly of actin filaments. Cell. 2003;112:453-65.

34. Nishita M, Tomizawa C, Yamamoto M, Horita Y, Ohashi K, Mizuno K. Spatial and temporal regulation of cofilin activity by LIM kinase and slingshot is critical for directional cell migration. J Cell Biol. 2005;171:349-59.

35. Petrilli A, Copik A, Posadas M, Chang LS, Welling DB, Giovannini M, Fernandez-Valle C. LIM domain kinases as potential therapeutic targets for neurofibromatosis type 2. Oncogene. 2014;33:3571-82.

36. Yoshioka K, Foletta V, Bernard O, Itoh K. A role for LIM kinase in cancer invasion. Proc Natl Acad Sci U S A. 2003:100:7247-52.

37. Amano T, Kaji N, Ohashi K, Mizuno K. Mitosis-specific activation of LIM motif-containing protein kinase and roles of cofilin phosphorylation and dephosphorylation in mitosis. J Biol Chem. 2002;277:22093-102.

38. Po'uha ST, Shum MS, Goebel A, Bernard O, Kavallaris M. LIM-kinase 2, a regulator of actin dynamics, is involved in mitotic spindle integrity and sensitivity to microtubule-destabilizing drugs. Oncogene. 2010;29:597-607.

39. Sumi T, Matsumoto K, Nakamura T. Mitosis-dependent phosphorylation and activation of LIM-kinase 1. Biochem Biophys Res Commun. 2002;290:1315-20.

40. Croft DR, Olson MF. The rho GTPase effector ROCK regulates cyclin a, cyclin D1, and p27Kip1 levels by distinct mechanisms. Mol Cell Biol. 2006;26:4612-27.

41. Sumi T, Hashigasako A, Matsumoto K, Nakamura T. Different activity regulation and subcellular localization of LIMK1 and LIMK2 during cell cycle transition. Exp Cell Res. 2006;312:1021-30.

42. Kaji N, Muramoto A, Mizuno K. LIM kinase-mediated cofilin phosphorylation during mitosis is required for precise spindle positioning. J Biol Chem. 2008; 283:4983-92.

43. Ahringer J. Control of cell polarity and mitotic spindle positioning in animal cells. Curr Opin Cell Biol. 2003;15:73-81.
44. Macara IG. Parsing the polarity code. Nat Rev Mol Cell Biol. 2004;5:220-31.

45. Calegari F, Huttner WB. An inhibition of cyclin-dependent kinases that lengthens, but does not arrest, neuroepithelial cell cycle induces premature neurogenesis. J Cell Sci. 2003;116:4947-55.

46. Lange C, Huttner WB, Calegari F. Cdk4/CyclinD1 overexpression in neural stem cells shortens $\mathrm{G} 1$, delays neurogenesis, and promotes the generation and expansion of basal progenitors. Cell Stem Cell. 2009;5:320-31.

47. Pilaz L, Patti D, Marcy G, Ollier E, Pfister S, Douglas RJ, Betizeau M, Gautier E, Cortay V, Doerflinger N, et al. Forced G1-phase reduction alters mode of division, neuron number, and laminar phenotype in the cerebral cortex. $\mathrm{P}$ Natl Acad Sci USA. 2009;106:21924-9.

48. Takahashi H, Koshimizu U, Miyazaki J, Nakamura T. Impaired spermatogenic ability of testicular germ cells in mice deficient in the LIM-kinase 2 gene. Dev Biol. 2002;241:259-72.

\section{Publisher's Note}

Springer Nature remains neutral with regard to jurisdictional claims in published maps and institutional affiliations.
Ready to submit your research? Choose BMC and benefit from:

- fast, convenient online submission

- thorough peer review by experienced researchers in your field

- rapid publication on acceptance

- support for research data, including large and complex data types

- gold Open Access which fosters wider collaboration and increased citations

- maximum visibility for your research: over $100 \mathrm{M}$ website views per year

At BMC, research is always in progress.

Learn more biomedcentral.com/submissions 